\title{
Surgical Treatment of Strabismus in Children
}

\author{
Alketa Tandili ${ }^{1}$, Sulejman Zhungli $^{2}$ \\ University Hospital Centre - Mther Theresa” Tirana, Albania
}

\begin{abstract}
Strabismus is a visual disorder in which the eyes are misaligned and point in different directions. This misalignment may be constant or intermittent. The aim of this study was to assess pediatric strabismus outcomes andcomplications in a tertiary hospital. The study was conducted at University Hospital Center "Mother Theresa" in Tirana, Albania over the period 2012 -2014.This study includes 82 cases, $49(60 \%)$ males and 33(40\%) females with mean age of $12.2( \pm 12.2)$ years. Exodeviation (51\%) and esodeviation(39\%) had more frequency than other deviation types. In all infantile types of strabismus, occurence age was less than 6 months.Male cases had more frequency of esotropia (55\%) and DRS II (78\%) than females while in other types females were more frequent (p<0.05).Twenty nine percent of cases had family history of strabismus. Congenital type of strabismus was the most common cause (68\%) of pediatric strabismus.
\end{abstract}

Keywords: pediatric strabismus, exodeviation, esodeviation, surgery

\section{Introduction}

Strabismus is a visual disorder in which the eyes are misaligned and point in different directions. This misalignment may be constant or intermittent. When the eyes are misaligned, typically one eye will fixate on objects of interest while the other eye turns in (esotropia), out (exotropia), down (hypotropia), or up (hypertropia) (1).Often times the eye that is fixing on objects switches; that is, the misaligned eye will fixate and the previously fixing eye will become the misaligned eye. This alternation of deviating eye is often a good sign suggesting that the vision in each eye is equal (2). On the other hand, if the eyes do not switch fixation (one eye is constantly the fixating eye and the other eye is constantly the misaligned eye), then the fixating eye is favored and almost always has better vision (3).Esotropia and exotropia are common conditions among children. Eye misalignment typically results in double vision in adults, but the developing brain in a child deals with the double vision by suppressing one of the images. Therefore, abnormal eye alignment in childhood blocks normal binocular vision development (as the brain learns to rely on only one image from the fixing eye) $(4,5)$.Although the avoidance of double vision is beneficial in some regard, this adaptation by the developing brain is also detrimental because the ignored eye loses the ability to see perfect 20/20" vision - a condition called amblyopia. Amblyopia is decreased vision in a perfectly healthy and well-formed eye which occurs because of a loss of the connection between an eye and the brain during a critical period of vision development from birth to 8 or 9 years of age $(6,7)$. Young patients with eye misalignment also typically have poor stereo or 3D vision and depth perception. Strabismus in adults often results in double vision because the brain has been trained to receive images from both eyes $(8,9)$. Adults with strabismus are not at risk of developing amblyopia because the connections between the eye and the brain are already formed and cannot be suppressed (10). The aim of this study was to assess pediatric strabismus outcomes andcomplications in a tertiary hospital.

\section{Material and Methods}

The study was conducted at University Hospital Center Mother Theresa" in Tirana, Albania.

Inclusion criteria of this study were: 1) Referral to our ophthalmology clinic with full medical records; 2) Onset age of strabismus below 16 years; 3) At least 6 months postoperative follow-up. Based on inclusion criteria, we analyzed 82 cases that were reported over the period 2012 2014. Data were extracted from questionnaires containing 13 questions. We assessed age of onset (month) and surgery ages (year),refractive errors (Spherical Equivalent $=$ Sphere $+1 / 2$ Astigmatism) and series of operations as quanititative and other variables including gender, strabismus family history, amblyopia, strabismus types and causes, mangement type and operation, outcomes and complications as qualitative variables. Based on direction, strabismus types are classified asesotropia, exotropia, hypertropia, hypotropia, inferior oblique overaction, superior oblique paralysis and duaneretraction syndrome (DRS). Causes of strabimus were calssified as congenital, accomodative, paralytic, mechanical, postoperative, traumatic, refractive and sensory deprivative. The strabismus mangements included: glasses, eye patching, surgery and follow-up. Operation techniques for different types of strabismus were: bilateral rectus (recess, resection), bimedial rectus (recess, resection), inferior oblique recess, recess and resection, superior rectus, inferior rectus (recess, resection) and superior oblique (tenotomy, tighthening).Successful outcome in our survey was defined as postoperative residual deviation to be less than 8 prism diopters. Misalignments were defined as undercorrection or overcorrection both with more than 8 prisms of deviation. Operation complications include residual deviation, overcorrection deviation, conjunctival infection, suture sensitivity, amblyopia, conjunctival dehiscence, diplopia, suture granuloma and dellen formation. Postoperative follow-up time was at least six months in this study.

\section{Results and Discussion}

This studyincludes 82 cases, 49 (60\%) males and 33(40\%) females with mean age of $12.2( \pm 12.2)$ years. Exodeviation 


\section{International Journal of Science and Research (IJSR) \\ ISSN (Online): 2319-7064}

Index Copernicus Value (2013): 6.14 | Impact Factor (2014): 5.611

$(51 \%)$ and esodeviation(39\%) had more frequency than other deviation types (fig. 1). In all infantile types of strabismus, occurence age was less than 6 months. Fisher exact test show that age of children had statistically significant association only with exotropic type with inferior oblique overaction and DRS $(\mathrm{p}<0.05)$. Surgery age in esotropia (59\%), hypertropia (48\%), hypotropia(67\%), inferior oblique overaction (56\%), DRS II (100\%) and combined type (55\%) was less than 10 years old. Operation age had signifiacnt association withoperation type in patients with esotropia, exotropia, inferior oblique overaction and combined type $(\mathrm{p}<0.05)$. Male cases had more frequency of esotropia $(55 \%)$ and DRS II $(78 \%)$ than females while in other types females were more frequent $(\mathrm{p}<0.05)$.

Twenty nine percent of cases had family history of strabismus. There was no significant association between strabismus occurrence and family history of patients $(p>0.05)$. Congenital type of strabismus was the most common cause $(68 \%)$ of pediatric strabismus (fig. 2).Ten percent of cases had amblyopia and $60 \%$ had refractive errors. In the right eye, we observed hypermetropia in $(47 \%)$, myopia in $(32 \%)$ and astigmatism in $(16 \%)$ cases. In the left eye hypermetropia (52\%), myopia (21\%) and 60 astigmatism (14\%) were seen. There was anisometropiain (11\%) of cases. Management of patients included: glasses, eye patching, surgery and observing. The most common complications of surgery were undercorrection and overcorrection. These complications might be due to surgery technique, individual character, reduced visual acuity, accommodation change or scar tissue stretching, but in many cases, the etiology was unknown (11). In astudy on 123 patients with intermittent exotropia, onset age, operation age, strabismus duration, amblyopia and anisometropia had no significant impacts on operation outcomes (12.The treatment goal for strabismus is to allow for normal vision development, realign the eyes, prevent amblyopia (lazy eye), and attempt to restore stereo or 3-dimensional vision. If amblyopia is detected in the first few years of life, treatment is often successful. If diagnosis and treatment is delayed until later, amblyopia (reduced vision in one or both eyes caused by disuse) may become permanent (13). Occlusive patching of the better sighted eye can force use of the amblyopic eye and improve vision in the amblyopic eye. Depending on the cause of the strabismus or amblyopia, treatment may involve repositioning the unbalanced eye muscles, removing a cataract, or correcting other conditions that are causing the eyes to turn. After a complete eye examination, refraction and detailed study of the inner parts of the eye, an ophthalmologist can recommend appropriate optical, medical, or surgical treatment (14).Early surgery is often recommended to correct strabismus in younger infants, who can then develop normal acuity and binocular (stereo) vision once the eyes are straightened. As a child gets older, the chance of developing normal sight and depth perception decreases. Crossed eyes can also have a negative effect on a child's coordination and motor skills, social interaction and self-confidence. Strabismus surgery involves making a small incision in the tissue covering the eye, which allows the ophthalmologist to access the underlying eye muscles (15).The eyeball is never removed from the socket during this kind of eye surgery. Which eye muscles are repositioned during the surgery and by how much depends upon detailed measurement of the type of strabismus. It may be necessary to perform eye muscle surgery on one or both eyes. When strabismus surgery is performed on children, a general anesthetic is required; a local anesthetic is often an option for adults. Eye muscle surgery is generally performed as an outpatient procedure in a hospital or a surgery center (16). Recovery time is rapid and the patient is usually able to resume normal activities within a few days. Following surgery, glasses or prisms may sometimes be needed to fine tune" the result. Over-or-under correction can occur and further "touch-up" surgery may be needed (17). As with any surgery, eye muscle surgery has certain risks which include over or under correction of the strabismus, infection, bleeding, excessive scarring, and other complications that very rarely may lead to loss of vision.

\section{Conclusion}

Strabismus surgery is a common procedure and most patients will see a large improvement in the alignment of their eyes after surgery. In some cases, they may need additional surgery or prism glasses to optimally align the eyes. Each case of strabismus is unique and should be discussed with physicins to understand the goals and expectations of surgery.

\section{References}

[1] Hertle, Richard. "Eye Muscle Surgery and Infantile Nystagmus Syndrome" (Microsoft Word document). American Nystagmus Network. Retrieved 2006-10-10.

[2] Gunter K. von Noorden: Binocular Vision and Ocular Motility: Theory and management of strabismus, Chapter 26: Principles of Surgical Treatment, telemedicine.orbis.org

[3] Surgery Encyclopedia - Eye Muscle Surgery

[4] Strabismus.com - Strabismus Surgery

[5] Parikh, RK; Leffler, CT (July 2013). "Loop suture technique for optional adjustment in strabismus surgery". Middle East African Journal of Ophthalmology 20 (3).

[6] Eye Procedures $>$ Adjustable Suture Strabismus Surgery - EyeMDLink.com

[7] Kushner BJ, Fisher M. Is alignment within 8 prism diopters of orthotropia a successful outcome for infantile esotropia surgery? Arch Ophthalmol. February 1996, Volume 114, Issue 2, pages 176-180. PMID 8573021

[8] Yagasaki, T.; Yokoyama, Y. O.; Maeda, M. (Jul 2011). "Influence of timing of initial surgery for infantile esotropia on the severity of dissociated vertical deviation". Jpn J Ophthalmol 55 (4): 383 388. doi:10.1007/s10384-011-0043-1. PMID 21647566.

[9] "Discussion" in: Pradeep Sharma; S. Thanikachalam; SachinKedar; Rahul Bhola (January-February 2008). "Evaluation of subjective and objective cyclodeviation following oblique muscle weakening procedures".Indian Journal of Ophthalmology 56 (1). pp. 39-43. doi:10.4103/0301-4738.37594. PMCID: PMC2636065.

[10]H. D. Schworm; S. Eithoff; M. Schaumberger; K. P. Boergen (February 1997). "Investigations on subjective and objective cyclorotatory changes after inferior

\section{Volume 5 Issue 1, January 2016}




\section{International Journal of Science and Research (IJSR) \\ ISSN (Online): 2319-7064}

Index Copernicus Value (2013): 6.14 | Impact Factor (2014): 5.611

oblique muscle recession.". Investigative Ophthalmology \& Visual Science 38 (2). pp. 405-412.

[11]Khawam, E.; Jaroudi, M.; Abdulaal, M.; Massoud, V.; Alameddine, R.; Maalouf, F. (2013). "Major review: Management of strabismus vertical deviations, A- and V-patterns and cyclotropia occurring after horizontal rectus muscle urgery with or without Oblique Muscle Surgery". Binocul Vis Strabolog Q Simms Romano 28 (3): 181-192. PMID 24063512.

[12] Guthrie, ME; Wright, KW (September 2001). "Congenital esotropia". OphthalmolClin North Am 14 (3): $\quad 419-24, \quad$ viii. doi:10.1016/S08961549(05)70239-X. PMID 11705141.

[13]Edelman PM (2010). "Functional benefits of adult strabismus surgery". Am Orthopt J. 60 (60): 4347. PMID 21061883.

[14] Kersey, J. P.; Vivian, A. J. (Jul-Sep 2008). "Mitomycin and amniotic membrane: a new method of reducing adhesions and fibrosis in strabismus surgery". Strabismus 16 (3): 116-
118. doi:10.1080/09273970802405493.PMID 18788060

[15] Wright, Kenneth W.; Thompson, Lisa S.; Strube, Yi Ning; Coats, David K. (August 2014). "Novel strabismus surgical techniques - not the standard stuff". Journal of American Association for Pediatric Ophthalmology and Strabismus (JAAPOS) 18 (4): e47. doi:10.1016/j.jaapos.2014.07.152.

[16]Kushner, Burton J. (2014). "The Benefits, Risks, and Efficacy of Strabismus Surgery in Adults". Optometry and Vision Science 91 (5): e102e109. doi:10.1097/OPX.0000000000000248. ISSN 104 0-5488.PMID 24739461.

[17] Engel JM (September 2012). "Adjustable sutures: an update". Current Opinion in Ophthalmology 23 (5): $373-$

6. doi:10.1097/ICU.0b013e3283567321. PMID 228718 79.

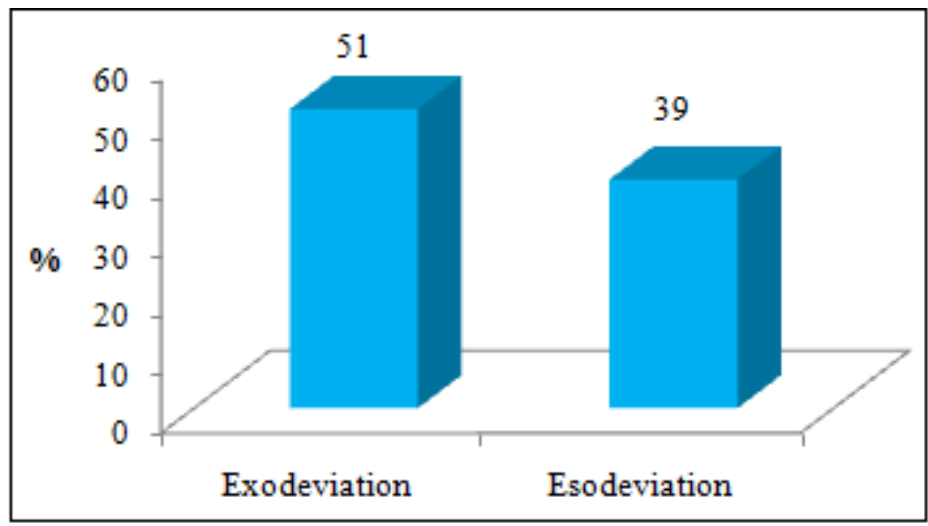

Figure 1: The type of eye deviation

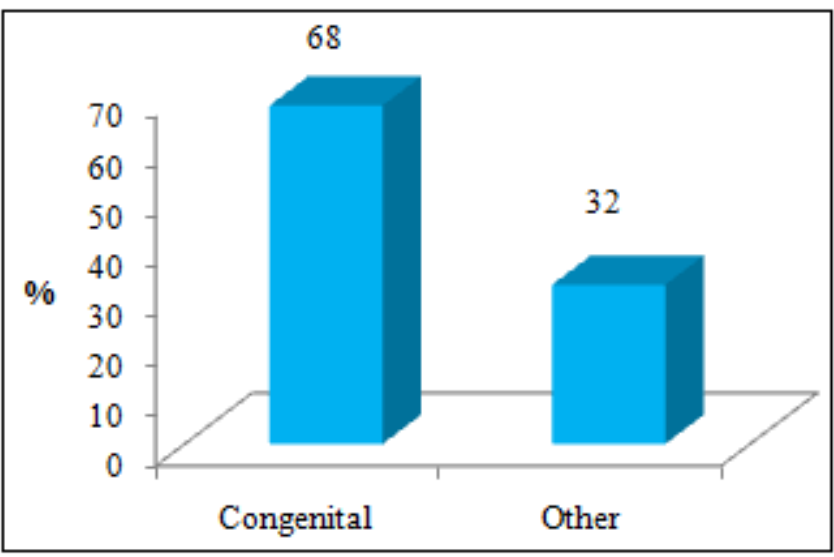

Figure 2: The cause of strabismus 